\title{
New arsenals for cancer
}

Keywords: regulatory hurdles, clinical trials, cancer, genetic markers, diagnostic

\section{Mini review}

Researchers are employing scientific learnings and new technologies to tackle the most complex and challenging diseases. There are several bizarre factors i.e. regulatory hurdles, longer and complex clinical trials etc are contributing to increasing costs, time, and uncertainty related to drug development. Expanding knowledge of the mechanisms underlying cancer has uncovered the immense complexity of the many forms of this disease but has also revealed new possible ways to attack cancer cells. Many researchers are now focusing on how to better categorize different cancers. Historically, a particular type of cancer was identified based on the tissue in which the cancer cells began to develop; however, researchers today are increasingly able to define different types of cancer based on biologic characteristics. These advances are enabling researchers to better combat cancer by targeting the root causes of the disease.

Oncology research has been transformed dramatically over the past several decades, with advanced development in biological sciences informing improved screening and the development of new personalized medicines which plays with diagnostic tools and deals with specific biological markers \& patient's own molecular profile which is responsible for his/her response to any medicine. Notably some small differences such as in formulation or dosing, impact adherence can also affect the way the medicine works for a particular patient. Currently certain advancements are being practiced above mentioned diagnostic tests personalized medicine segment

a. Genetic markers (Certain no. of gene panel) which scan throughout the human genome with a patient's family and clinical history to predict their $\mathrm{N}$-year and lifetime risk of developing cancer.

b. Some test involves the combination of genetic and clinical data to identify patient with low-risk cancer who can safely forego other therapies after surgical procedures.

c. Some diagnostic test that detects specific mutations and helps indicate whether or not patients with cancer who may preferentially benefit from the some inhibitor class of drugs.

d. Tests assess a cancer's inability to repair DNA damage. And patients benefit from DNA-damaging medicines and certain inhibitors.

So by administrating personalized medicines, specific pathways can be targeted, causing different types of cancer. Biologics represents another consortium of anticancer agents including nucleic acid, peptides and proteins. A brief introduction to such Biologics is mentioned below.

\section{Antibody drug conjugates}

Antibodies are immune system proteins that recognize specific targets for the immune system to attack, such as viruses, bacteria, or tumor cells. Antibody drug conjugates are particularly complex as they require the combination of antibody, linkage agent, and a drug. The antibody targets and binds to particular proteins or receptors on the

\author{
Volume 7 Issue 2 - 2018
}

\author{
Sumeet Kapoor,' Amit K Gupta, ${ }^{2}$ Swati Ojha ${ }^{3}$ \\ 'Centre for Biomedical Engineering, Indian Institute of \\ Technology Delhi, India \\ ${ }^{2}$ Department of Mechanical Engineering, Indian Institute of \\ Technology Delhi, India \\ ${ }^{3}$ Department of Biochemical Engineering and Biotechnology, \\ Indian Institute of Technology Delhi, India
}

Correspondence: Sumeet Kapoor, Centre for Biomedical Engineering, Indian Institute of Technology Delhi, India, Email s.kapooriitd@gmail.com

Received: August 25, 2017 | Published: April 09, 2018

surface of a cancer cell and the chemotherapy is then directly targeted to the cancer cell. This delivery system is to target the specific cancer cells only and ensure the safety of other surrounding healthy cells.

\section{Therapeutic cancer vaccines}

Therapeutic vaccines treat an existing cancer by strengthening the body's natural defenses to fight with cancers. In simplified descriptionour immune system has 'brakes' that can be triggered by cancer cells and such brakes disable the system's ability to attack the destructive cells and save the body from them.

\section{DNA based anticancer therapies}

These conjugates can be designed to target mutated genes expressed in various cancer cells. Some of the commonly studied nucleic acids are TNF- $\alpha$, Bcl12-siRNA, MRP1-siRNA, VEGF-siRNA ${ }^{1,2}$ etc. as anticancer therapeutics. It generally involves sequencing the Nucleic Acid i.e. DNA and RNA of a patient's tumor which has been extracted from the routine histology sample used for diagnosis. The sequencing of DNA and RNA identifies genetic mutations specific to an individual's tumor and can be used to design a DNA based personalized medicine.

\section{Peptides}

Many therapies are currently being used to treat deadly cancer but over the years, peptides have been evolved as promising therapeutic agents. Peptides can also be designed to target a specific intracellular pathway leading to induction of apoptosis in cancer cell. Some of the examples of such peptides are $\mathrm{BH}_{3} / \mathrm{BCL}_{2}$ targeting peptides, ${ }^{3}$ luteinizing hormone-releasing hormone gene receptors (LHRHR) peptides and RGD peptides. ${ }^{4,5}$ Peptides are being used as: direct anticancer drugs, cytotoxic drug carriers, vaccines, hormones and radionuclide carriers. Peptide-based therapy can be mainly divided into 3 parts.

Peptide only therapy: It may specifically enhance the immune system's response to kill tumor cells. 
Peptide vaccines: Vaccine route have been used in advanced cancers to improve patients overall survival.

Peptide conjugated nanomaterial's: The combination of peptides with nanomaterials expands the therapeutic ability of peptides to treat cancer by enhancing drug delivery and sensitivity. Biodegrdable polymers for delivery of the biologics have been reported and emerging as potential alternates for existing chemos. ${ }^{6}$

\section{References}

1. Mishra S, Webster P, Davis ME. PEGylation significantly affects cellular uptake and intracellular trafficking of non-viral gene delivery particles. Eur J Cell Biol. 2004;83(3):97-111.

2. Remaut K, Lucas B, Braeckmans K, et al. Pegylation of liposomes favours the endosomal degradation of the delivered phosphodiester oligonucleotides. J Control Release. 2007;117:256-266.
3. Kolluri SK, Zhu X, Zhou X, et al. A Short Nur77-Derived Peptide Converts Bcl-2 from a Protector to a Killer. Cancer Cell. 2008;14(4):285-298.

4. Wang F, Li Y, Shen Y, et al. The functions and applications of RGD in tumor therapy and tissue engineering. Int J Mol Sci. 2013;14(7):1344713462 .

5. Laakkonen P, Porkka K, Hoffman JA, et al. A tumor-homing peptide with a targeting specificity related to lymphatic vessels. Nat Med. 2002;8(7):751-755.

6. Kumar M, Gupta D, Singh G, et al. Novel polymeric nanoparticles for intracellular delivery of peptide cargos: Antitumor efficacy of the BCL-2 conversion peptide NuBCP-9. Cancer Res. 2014;74(12):3271-3281. 